\title{
REMOÇÃO DE METAIS UTILIZANDO BIOSSURFACTANTE PRODUZIDO PELA PSEUDOMONAS AERUGINOSA ATCC 10145
}

\author{
L. C. FERREIRA ${ }^{1}$, M. E. M. G. DIAS ${ }^{1}$ e U. C. FILHO $^{1}$ \\ ${ }^{1}$ Universidade Federal de Uberlândia, Faculdade de Engenharia Química \\ E-mail para contato: larysse_caixeta@hotmail.com, maria.eduarda1996@ hotmail.com
}

\begin{abstract}
RESUMO - A poluição de ambientes aquáticos por metais pesados ocorre principalmente pelas atividades industriais e acidentes ambientais. Esses metais apresentam características que os tornam nocivos aos organismos vivos. Dessa forma, a emulsão de biossurfactante surge como uma técnica de remoção desses compostos. O biossurfactante produzido pela Pseudomonas aeruginosa buscou avaliar a remoção de manganês, zinco e ferro a partir de diferentes emulsões. Para o metal zinco esse processo não apresentou remoção, porém para o manganês apresentou $5 \%$ de remoção e para o ferro resultou em valores mais satisfatórios de aproximadamente $60 \%$.
\end{abstract}

\section{INTRODUÇÃ̃O}

A poluição ambiental em ambientes aquáticos por metais tóxicos é uma questão preocupante que se expande mundialmente com o crescimento dos processos de industrialização e urbanização (AHMAD et al., 2015, ISLAM et al., 2015, SINGH \& KUMA, 2017). Em países com atividade industrial avançada como os EUA, países europeus, Taiwan, India, China e Brasil, a presença de metais tóxicos ocorre, principalmente, pelo despejo de efluentes industriais e em decorrência de acidentes ambientais. Um exemplo de acidente ocorrido, foi o de 2015, no Brasil, em que uma mineradora, foi responsável pela liberação de elevadas concentrações de metais pesados como ferro e manganês em águas superficiais do país (MAITY et al., 2013, SEGURA et al., 2016)

As características de toxicidade, abundância e não biodegradabilidade dos metais pesados, faz com que esses compostos, se lançados em águas superficiais, ocasionem efeitos nocivos nos organismos vivos, ao bioacumularem ao longo da cadeia alimentar, fazendo com que esses compostos sejam removidos desses ambientes. Dentre as tecnologias desenvolvidas para a remoção de metais pesados, destaca-se o processo de remediação, que utiliza de compostos de origem microbiana para o tratamento dos ambientes contaminados (VENKATESWARAN et al., 2007, BELKHIRI et al., 2017, COMBY et al, 2014, KANOUN-BOULE et al., 2009).

Diversos processos de remediação de ambientes contaminados com metais pesados, utilizam compostos de origem microbiana denominados de biossurfactantes. Esses compostos são moléculas com propriedades tensotivas resultantes das atividades metabólicas de fungos, leveduras e bactérias (LUNA et al., 2016, SILVA et al., 2010). 
O presente trabalho busca investigar o uso de emulsão de biossurfactante produzido pela Pseudomonas aeruginosa na presença de EDTA como agente complexante de metais ferro, zinco e manganês.

\section{MATERIAL E MÉTODOS}

\subsection{Microrganismo e fermentação}

A produção de biossurfactante foi avaliada utilizando o microrganismo Pseudomonas aeruginosa ATCC 10145 fornecida pela Coleção de Culturas Tropicais da Fundação Tosello, (Campinas-SP). Para a caracterização do surfactante produzido foram realizados os testes de concentração de biomassa, concentração de surfactante, índice de emulsificação e tensão superficial ao longo da fermentação de 0 a 120 horas. Essa fermentação com o microrganismo foi realizada com o meio descrito por Sarubbo et al (2010), composto por 30,0 g/L de glicerol, 6,0 de $\mathrm{NaNO}_{3}, 1,0 \mathrm{~g} / \mathrm{L}$ de $\mathrm{KH}_{2} \mathrm{PO}_{4}, 1,0$ de $\mathrm{K}_{2} \mathrm{HPO}_{4}, 0,2$ de $\mathrm{MgSO}_{4} .7 \mathrm{H}_{2} \mathrm{O}, 0,02$ de $\mathrm{CaCl}_{2} .2 \mathrm{H}_{2} \mathrm{O}$ e 0,05 de $\mathrm{FeCl}_{3} \cdot 6 \mathrm{H}_{2} \mathrm{O}$.

\subsection{Remoção de metais}

A remoção dos metais ferro, zinco e manganês foi avaliada em emulsão contendo EDTA e $\mathrm{NaCl}$ e meio fermentado por 72 horas nas condições descritas na Tabela 1.

Tabela 1 - Condições das emulsões.

\begin{tabular}{|c|c|c|c|c|}
\hline Nomenclatura & EDTA $(\mathrm{mM})$ & $\mathrm{NaCl}$ & $\mathrm{HCl}$ & Surfactante $(\mathrm{g} / \mathrm{L})$ \\
\hline \hline A-E10-S2-m & 10 & $2 \%$ & $0,7 \%$ & 0,0745 \\
\hline A-E10-S2 & 10 & $2 \%$ & $0,7 \%$ & 0,1490 \\
\hline A-E10-S0-m & 10 & $0 \%$ & $0,7 \%$ & 0,0745 \\
\hline A-E10-S0 & 10 & $0 \%$ & $0,7 \%$ & 0,1490 \\
\hline A-E0-S2-m & 0 & $2 \%$ & $0,7 \%$ & 0,0745 \\
\hline A-E0-S2 & 0 & $2 \%$ & $0,7 \%$ & 0,1490 \\
\hline A-E0-S0-m & 0 & $0 \%$ & $0,7 \%$ & 0,0745 \\
\hline A-E0-S0 & 0 & $0 \%$ & $0,7 \%$ & 0,1490 \\
\hline
\end{tabular}

\subsection{Metodologia de quantificação}

A remoção de manganês foi avaliada por absorção atômica em chamas da marca SHIMADZU (modelo AA-7000). Após essa leitura a remoção foi obtida pelo cálculo da Equação 1, em que Ci é a concentração inicial de manganês e Cf a concentração final:

$$
\operatorname{Remoção~}(\%)=\frac{c_{\mathrm{i}}-c_{f}}{c_{\mathrm{i}}} \times 100
$$

\section{RESULTADOS E DISCUSSÃO}

O primeiro metal no estudo da remoção foi o zinco, o qual não apresentou remoções em nenhuma condição de emulsão estudada. A Figura 1 apresenta os dados de remoção para metal manganês em que para o tempo de contato de 5 minutos apresentou uma remoção de 
aproximadamente 4\% na condição A-E0-S2-m, com ausência de remoção nas demais condições estudadas.

Figura 1 - Gráfico de remoção do manganês.

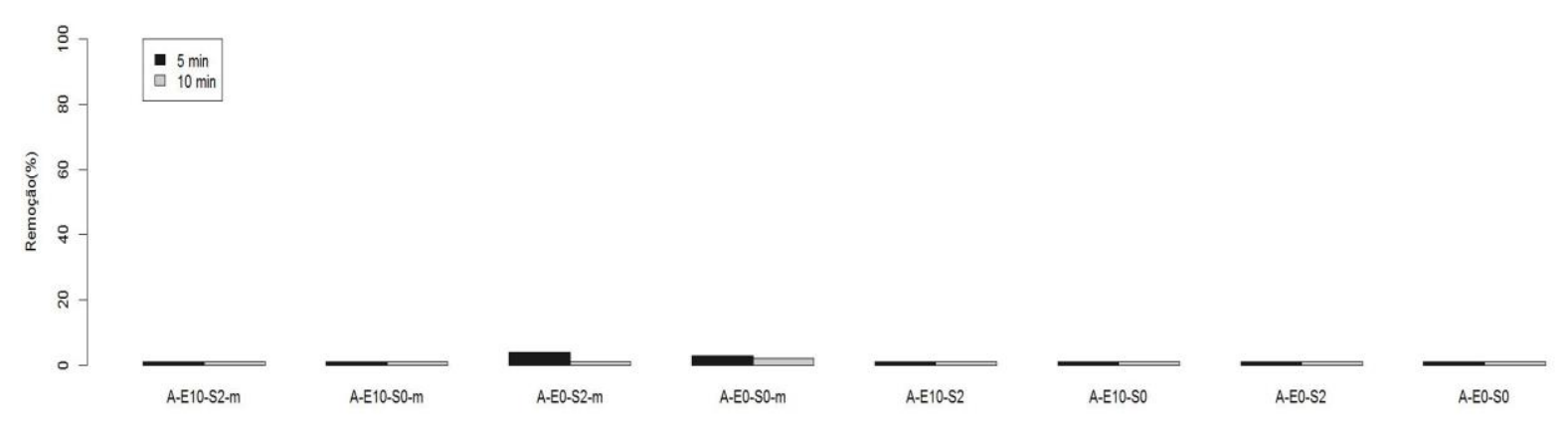

A Figura 2 representa o gráfico com os resultados de remoção do ferro. Diferentemente dos metais zinco e manganês, o ferro apresentou remoções satisfatórias nas condições de emulsão avaliadas. Verifica-se que em relação ao tempo de contato da emulsão com o metal, o período de 5 minutos resultou em remoções mais satisfatórias. Em relação às condições estudas de emulsão observa-se que a condição A-E10-S2 apresentou remoções de aproximadamente $30 \%$ para os tempos de 5 e 10 minutos. As condições A-E0-S0-m, A-E0-S2 e A-E0-S0 resultaram em remoções mais satisfatórias, atingindo um valor de aproximadamente $60 \%$.

Figura 2 - Gráfico de remoção do ferro.

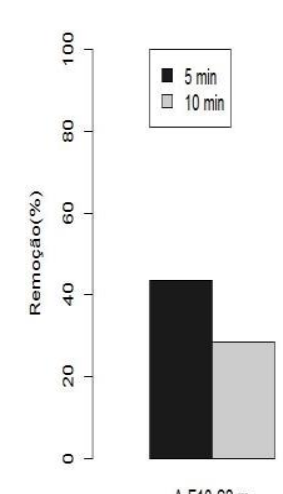

A.E10-52-m

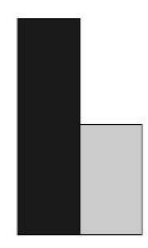

A-E10-S0-m

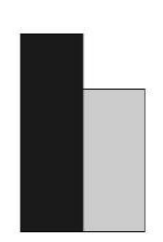

A-E0-52-m

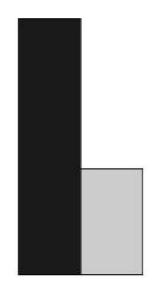

A-EO-SO-m

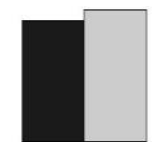

A-E10-S2

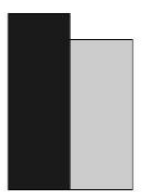

A-E10-SO

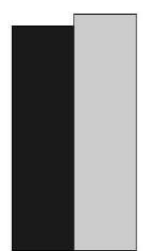

A-E0-S2

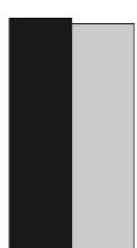

A-EO-SO

A diferença de remoção entre os metais zinco, ferro e manganês pode ser explicada parcialmente pela própria natureza do mecanismo de remoção da membrana do biossurfactante.

Na membrana, o EDTA é responsável pela remoção e a faz por ação quelante nos íons metálicos. Para os íons $\mathrm{Zn}^{2+}, \mathrm{Fe}^{3+}$ e $\mathrm{Mn}^{2+}$, segundo HARRIS (1999), as constantes de afinidade ou constantes de formação de complexo metal-EDTA, são respectivamente 16,50, 
25,1 e 13,87. Pode-se observar que a remoção esperada em ordem de maior para menor corresponde a ferro, zinco e manganês. A comparação das remoções com as constantes justifica o fato de o ferro ser o metal com maior remoção observada, mas não justifica o fato da remoção do zinco ser inferior à do manganês. Isso ocorre porque os valores das constantes de afinidade referem-se ao EDTA na forma totalmente ionizada, o que, segundo HARRIS (1999), não ocorre em pH inferior a 10,24. Neste contexto, vale ressaltar que os resultados das Figuras 1 e 2 referem-se a meio ácido $(0,7 \%$ de $\mathrm{HCl}$, como mostra a Tabela 1$)$, pois o uso de condições ótimas para o EDTA favorecem a precipitação, ou seja, geram a remoção por dois mecanismos, ao invés de seguir o mecanismo associado a membranas, que representa a proposta seguida neste trabalho.

\section{CONCLUSÃO}

A bactéria Pseudomonas aeruginosa demonstrou ser produtora de biossurfactante com características promissoras na remoção de ferro. Além disso, a partir dos experimentos realizados, nas diferentes condições foi possível concluir que, o processo de remoção pela formação da emulsão de biossurfactante demonstrou ser um processo eficaz, ao verificar uma remoção de $60 \%$ de ferro.

\section{REFERÊNCIAS}

AHMAD, A. L.; SHAH BUDDIN, M. M. H.; Ooi, B. S . Extraction of Cd (II) ions by emulsion liquid membrane (ELM) using Aliquat 336 as carrier. American Jornal of Chemistry, v. 5, p. 1-6, 2015.

BELKHIRI, L.; MOUNI, L.; NARANY, T. S.; TIRI, A. Evaluation of potencial health risk of heavy metals in groundwater using the integration of indicator kringing and multivariate statistical methodos. Groundwater for Sustainable Development, v.4, p. 12-22, 2017.

COMBY, E.; LE LAY, Y-F.; PIÉGAY, H. How chemical pollution becomes a social problem. Risk communication and assessment through regional newspapers during the management of PCB pollution of the Rhône River (France). Science Total Environmental, v. 482-483, p.100-115, 2014.

HARRIS, D. C. Quantitative Chemical Analysis. W. H. Freeman and Company, v.1, p. 265266, 1999.

ISLAM, M. S.; AHMED, M. K.; RAKNUZZAMAN, M.; HABIBULLAH-AL-MAMUN, M.; ISLAM, M. K. Heavy metal pollution in surface water and sediment: a preliminary assessment of an urban river in a developing country. Ecol. Indic., v. 48, p. 282-291, 2015.

KANOUN-BOUlÉ, M.; VICENTE, J. A. F.; NABAIS, C.; PRASAD, M. N. V AND FREITAS, H. Ecophysiological tolerance of duckweeds exposed to copper. Journal Aquatic Toxicology., v.91, p.1-9, 2009. 
LUNA, J. M.; RUFINO, R. D.; SARUBBO, L. A. Biosurfactant from Candida sphaerica UCP0995 exhibiting heavy metal remediation proerties. Process Safety and Environmental Protection, v. 102, p. 558-566, 2016.

MAITY, J.P.; HUANG, Y.M.; HSU, C-M.; WU, C-I.; CHEN, C-C.; LI, C-Y.; JEAN, J-S.; CHANG, Y-F.; CHEN, C-Y. Removal of $\mathrm{Cu}, \mathrm{Pb}$ and $\mathrm{Zn}$ by foam fractionation and a soil washing process from contaminated industrial soils using soapberry-derived saponin: a comparative effectiveness assessment. Journal Chemosphere, v. 92, p.1286-1293, 2013.

SEGURA, F. R.; NUNES, E. A.; PANIZ, F. P.; PAULELLI, AC. C.; RODRIGUES, G. B.; BRAGA, G. U. L.; FILHO, W. D. P.; JR, F. B.; CERCHIARO, G., SIVA, F. F.; BATISTA, B. L. Potential risks of the residue from Samarco's mine dam burst (Bento Rodrigues, Brasil). Environmental Pollution, v. 218, p. 813-825, 2016.

SILVA, S. N. R. L., FARIAS, C. B. B., RUFINO, R. D., LUNA, J. M., SARUBBO, L. A. Glycerol as substrate for the production of biosufactant by Pseudomonas aeruginosa UCP0992. Colloids and Surfaces B: Biointerfaces, v. 79, p. 174-183, 2010.

SINGH, U. K.; AND KUMAR, B. Pathways of heavy metals contamination and associated human health risk in Ajay River basin, India. Chemosphere, v. 174, p. 183-199, 2017.

VENKATESWARAN, P.;VELLAICHAMY, S.; PALANIVELU, K. Speciation of heavy metals in electroplating industry sludge and wastewater residue using inductively coupled plasma. Int. J. Environ. Sci. Technol, v.4, p. 497-504, 2007. 